\title{
Infective Endocarditis with Intermittent Atrioventricular Block and Pseudoaneurysm of the Mitral-aortic Intervalvular Fibrosa in a Patient with Severe Aortic Stenosis
}

\author{
Hideyuki Hasebe ${ }^{1}$, Akira Takanohashi ${ }^{2}$, Kazuaki Shirota ${ }^{2}$ and Hajime Nakamura ${ }^{2}$
}

\begin{abstract}
Atrioventricular (AV) block and pseudoaneurysm of the mitral-aortic intervalvular fibrosa (P-MAIVF) are rare complications of infective endocarditis (IE). A 72-year-old man with severe aortic stenosis was hospitalized due to IE. After admission, intermittent AV block and P-MAIVF were noted. Interestingly, an accelerated junctional rhythm was observed during the process of AV block resolution. Elective surgery, which included patch closure of the fistula and replacement of the aortic valve, was successfully performed. The patient has remained in good condition without the recurrence of $\mathrm{AV}$ block. This case report describes a rare comorbidity of AV block and P-MAIVF in a patient with aortic valve IE.
\end{abstract}

Key words: endocarditis, atrioventricular block, pseudoaneurysm of the mitral-aortic intervalvular fibrosa

(Intern Med 55: 2825-2829, 2016)

(DOI: 10.2169/internalmedicine.55.7031)

\section{Introduction}

There are various periannular complications associated with infective endocarditis (IE) of the aortic valve (1). Among them, atrioventricular (AV) block and pseudoaneurysm of the mitral-aortic intervalvular fibrosa (P-MAIVF) are rare and can be life-threatening (2-4). The present report describes a case of a 72-year-old man with Streptococcus sanguinis IE of the aortic valve. This is the first report to describe the comorbidity of intermittent $\mathrm{AV}$ block and PMAIVF in a patient with IE.

\section{Case Report}

A 72-year-old man was admitted to our institution with a 3-day history of a sustained high fever. He had undergone dental care, not including tooth extraction, without antibiotic prophylaxis 3 months before hospital admission. At the time of admission, the patient was receiving medical treatment for hypertension (amlodipine $5 \mathrm{mg}$ daily, valsartan $160 \mathrm{mg}$ daily) and diabetes mellitus. Physical findings revealed a height of $165 \mathrm{~cm}$, a weight of $50 \mathrm{~kg}$, a blood pressure of $98 / 60 \mathrm{mmHg}$, a regular pulse of 80 beats per minute (bpm) and a temperature of $39.6^{\circ} \mathrm{C}$. There were no rales in the chest, however, a grade 4 systolic murmur was audible in the right upper sternal border. Neither Osler's nodes nor Janeway lesions was observed. Laboratory testing revealed a WBC count of $13.5 \times 10^{9} / \mathrm{L}$ ( $86 \%$ neutrophils), a hemoglobin concentration of $10.9 \mathrm{~g} / \mathrm{dL}$, a C-reactive protein (CRP) level of $9.7 \mathrm{mg} / \mathrm{dL}$, a procalcitonin level of $1.06 \mathrm{ng} / \mathrm{mL}$, a blood sugar of $172 \mathrm{mg} / \mathrm{dL}$, and a hemoglobin A1c of 8.1\%. Electrocardiography revealed sinus rhythm, first-degree $\mathrm{AV}$ block with a PQ interval of 0.22 seconds, and left ventricular hypertrophy (Fig. 1A). All four sets of blood culture grew Streptococcus sanguinis. Transthoracic (TTE) and transesophageal echocardiography (TEE) revealed severe aortic stenosis (AS) with a calcified trileaflet. The estimated aortic valve area was $0.45 \mathrm{~cm}^{2}$, and the maximal and mean pressure gradient was 108 and $64 \mathrm{mmHg}$, respectively. No apparent vegetative masses were observed, however, the aortic valve ring was edematous and thickened. Although vegetative masses were not apparent, a diagnosis of possible IE was made according to one major modified Duke criterion

${ }^{1}$ Division of Arrhythmology, Shizuoka Saiseikai General Hospital, Japan and ${ }^{2}$ Division of Thoracic and Cardiovascular Surgery, Shizuoka Saiseikai General Hospital, Japan

Received for publication December 22, 2015; Accepted for publication February 22, 2016

Correspondence to Dr. Hideyuki Hasebe, h153478@siz.saiseikai.or.jp 

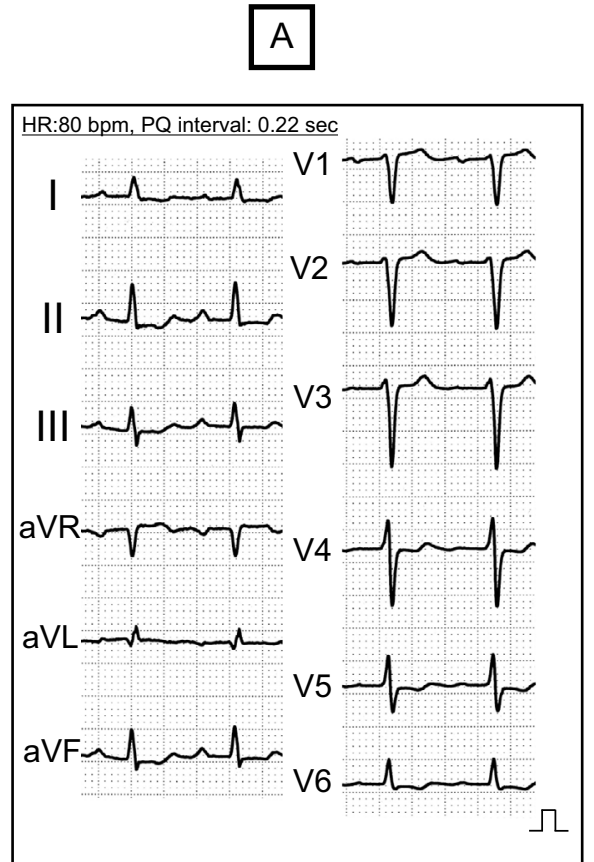
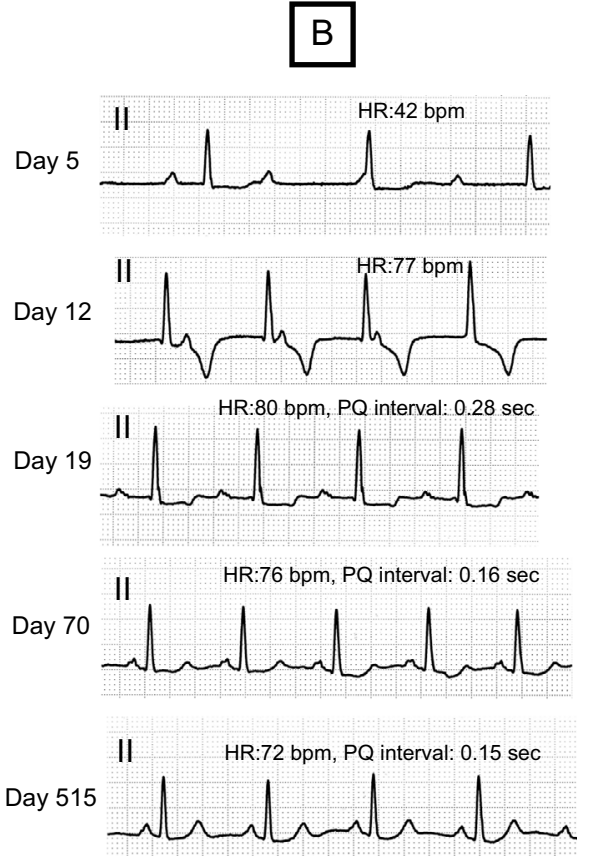

Figure 1. Results of a 12-lead electrocardiogram on admission (A) and the time course change in the electrocardiogram (B). An electrocardiogram at admission showed sinus rhythm, first-degree atrioventricular (AV) block and left ventricular hypertrophy. On hospital day 5, complete AV block was observed. An accelerated junctional rhythm (AJR) dissociated with atrial rhythm was subsequently seen on hospital day 12 . The heart rhythm changed from AJR to sinus rhythm with first-degree AV block on hospital day 19. The PQ interval gradually shortened with time.

(positive blood culture) and two minor criteria (predisposing AS, high fever). Intravenous administration of ceftriaxone (1 g every 12 hours) was started and continued till hospital day 4 as empiric antibacterial therapy. On hospital day 4, the causative organism was confirmed to be Streptococcus sanguinis, therefore antibacterial therapy was changed to intravenous daily administration of penicillin $G$ potassium $(24 \times$ $10^{6}$ unit) and gentamicin ( $1 \mathrm{mg} / \mathrm{kg}$ every 12 hours). These treatments resulted in defervescence and decreases in the WBC count, the CRP level, and the procalcitonin level.

On hospital day 5, the patient complained of dyspnea due to congestive heart failure (CHF). Electrocardiography showed complete AV block (Fig. 1B), which was the most likely cause of CHF. A transvenous pacing lead was immediately placed at the right ventricular apex for backup pacing at a rate of 60 beats per minute. The heart rhythm changed from complete AV block to an accelerated junctional rhythm (AJR) at a rate of $77 \mathrm{bpm}$ on hospital day 12 . The AJR persisted until hospital day 19, at which point electrocardiography showed sinus rhythm and first degree AV block with a PQ interval of 0.28 seconds (Fig. 1B). The patient maintained stable with sinus rhythm since that time, and the intravenous pacing lead was removed on hospital day 21. An electrophysiological study performed on hospital day 41 revealed an AH interval of $162 \mathrm{~ms}$, an HV interval of $58 \mathrm{~ms}$, and a Wenckebach rate of $200 \mathrm{bpm}$. Conduction block between His potential and ventricle was not induced. The clinical course was satisfactory except for heart failure due to AV block.

On hospital day 28, a pulsatile echo-free space between the anterior mitral leaflet (AML) and the aortic valve was newly detected by follow-up TTE and TEE (Fig. 2A and B). The echo-free space dilated during systole and collapsed during diastole, resulting in a pulsatile motion. Multidetector computed tomography (MDCT) performed on hospital day 29 showed an aneurysm-like structure over the sinus of Valsalva. Echocardiography with color Doppler was unable to determine whether there was a communication between the echo-free space and the adjacent structure. The communication site around the aneurysm-like structure was unclear on MDCT, and the three-dimensional MDCT image was not useful to clarify the locational relationship between the aneurysm-like structure and coronary artery (Fig. 2D). Therefore, left ventriculography (LVG) at the outflow tract was performed on hospital day 41. The contrast medium was seen entering from the LV outflow tract (LVOT) into the space between the left atrium (LA) and the sinus of Valsalva during systole and returning to the LVOT during diastole without any communication with other adjacent structures (Fig. 2C). Sinus of Valsalva aneurysm was denied, and a diagnosis of P-MAIVF was made. Elective surgery, including P-MAIVF repair and aortic valve replacement using a bioprosthetic valve, was performed on hospital day 55. During the surgery, a fistula was detected connecting the LVOT just below the non-coronary aortic cusp and the pseudoaneurysm (Fig. 3), and this fistula was closed with a patch. 


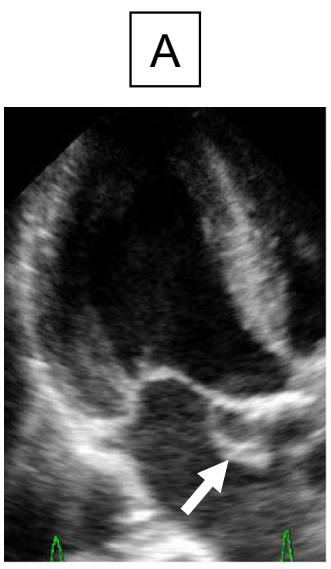

Systole

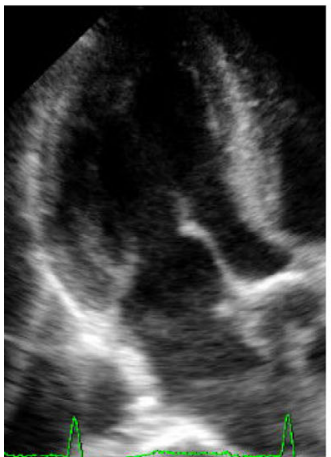

Diastole
B

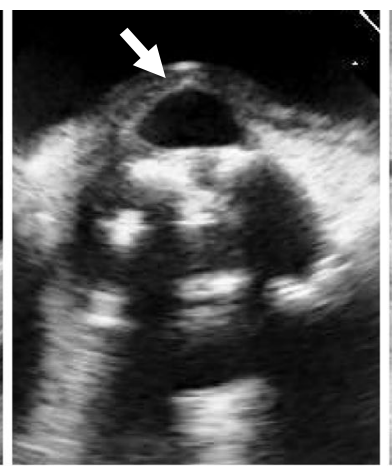

Systole

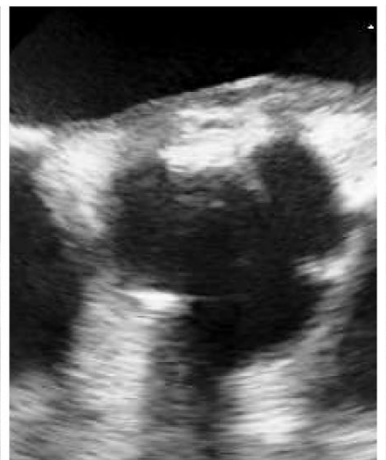

Diastole
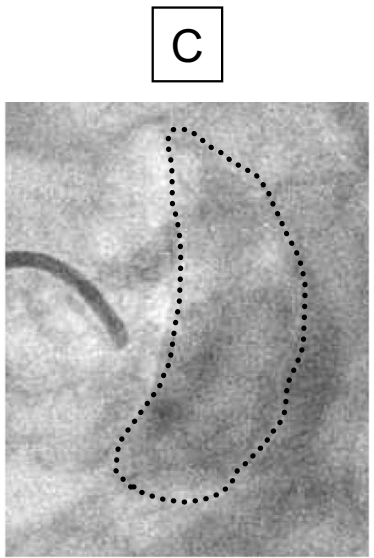

Systole

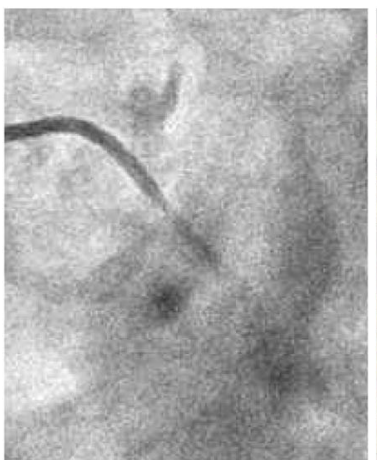

Diastole
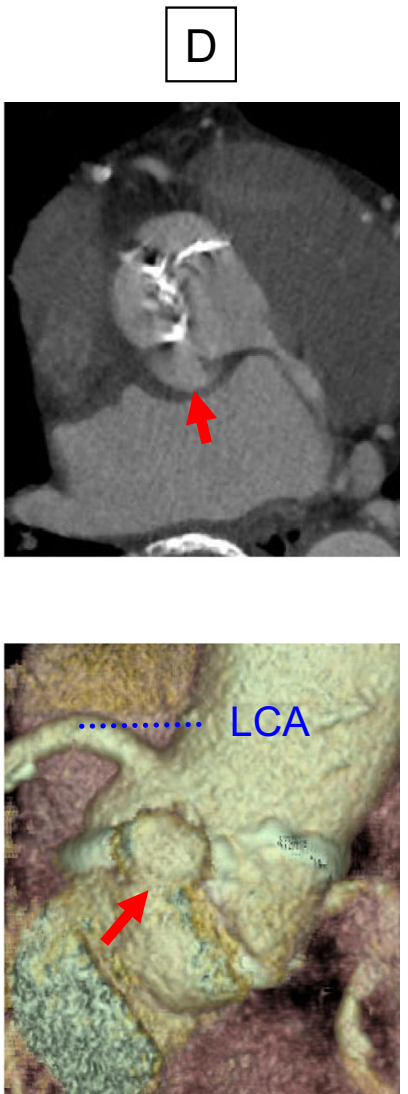

RAO $160^{\circ}$

Figure 2. Transthoracic echocardiography on hospital day 28 (A), transesophageal echocardiography on hospital day 29 (B), left ventriculography at the outflow tract on hospital day 41 (C), and multidetector computed tomography on hospital day 29 (D). The echo-free space dilated during systole (white arrow) and collapsed during diastole, resulting in pulsatile motion (A, B). The contrast medium was seen entering from the left ventricular outflow tract (LVOT) into the space between the left atrium and sinus of Valsalva during systole (surrounded by the black broken line) (C). The pseudoaneurysm was indicated by red arrows. Multidetector computed tomography was useful to clarify the locational relationship between the pseudoaneurysm and coronary artery (D). LCA: left coronary artery, RAO: right anterior oblique

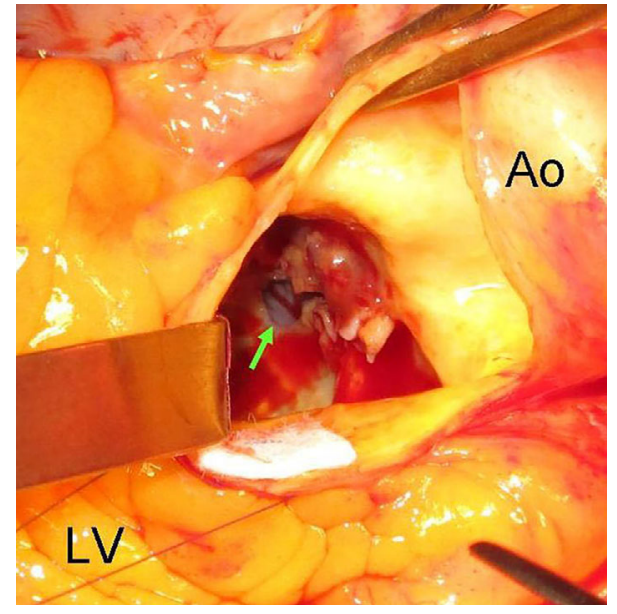

Figure 3. Intraoperative image. The root of the ascending aorta was opened and both non-coronary and left coronary cusps were resected, revealing the fistula in the non-coronary sinus of Valsalva (green arrow). Ao: ascending aorta, LV: left ventricle
There were no apparent vegetations, however, brown discoloration of the tissue around the fistula was noted, suggesting a recent infection. The patient's postoperative course was uneventful, and he was discharged on hospital day 70. Since then, he has remained in stable condition with no recurrence of infection or AV block for 16 months. The PQ interval shortened (0.16 seconds at discharge, 0.15 seconds at the last follow-up of day 515 from admission) with time.

\section{Discussion}

IE remains a dangerous condition, especially when it is associated with periannular extension (1). Among periannular complications, P-MAIVF is rare but potentially lifethreatening $(2,4)$. Another periannular complication of IE is AV block, which can be caused by IE involvement of the AV conduction system $(1,3,5)$. In the present case, both PMAIVF and AV block occurred during the subacute phase of IE. The comorbidity of P-MAIVF and AV block in a pa- 


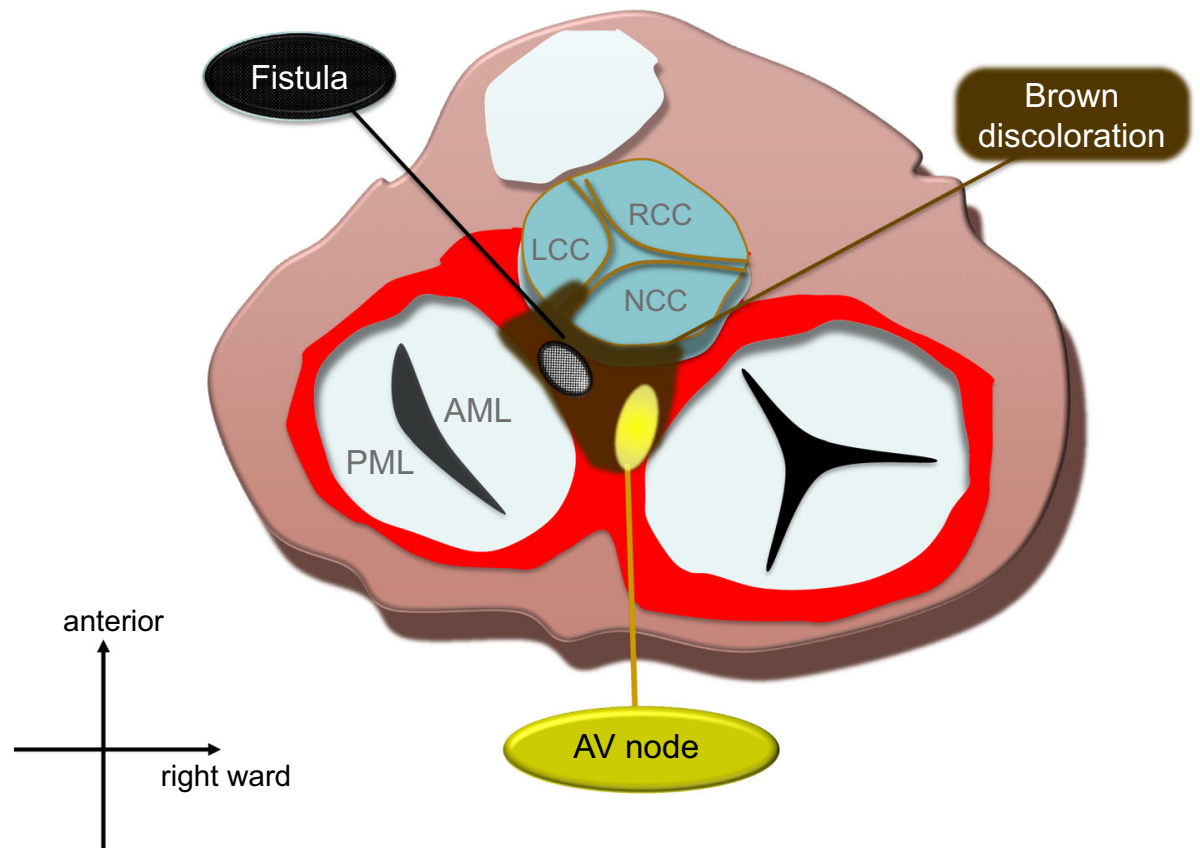

Figure 4. A schematic diagram representing the estimated locational relationship among the fistula, brown discoloration, and atrioventricular node. There was a fistula in the non-coronary sinus of Valsalva. Brown discoloration was detected in the tissue around the fistula. The atrioventricular node might have been involved in the discoloration area. AML: anterior mitral leaflet, AV node: atrioventricular node, LCC: left coronary cusp, NCC: non-coronary cusp, PML: posterior mitral leaflet, RCC: right coronary cusp

tient with IE is quite rare, and this is the first case report to describe this phenomenon.

P-MAIVF is a pseudoaneurysm of the inter-annular zone between the mitral and aortic valves and its communication with an LVOT. Endocarditis and aortic valve surgery are the two most frequent causes. The area of fibrous continuity between the aortic and mitral valves, described as MAIVF, is involved in the anatomical and functional integrity of the two valves. The MAIVF is histologically fibrous and avascular and is equivalent to the mitral valve leaflet structure. Because the MAIVF is avascular, infection of this tissue may result in abscesses or pseudoaneurysms or in direct communication between the LVOT and the LA (4). In the present case, the echocardiographic studies demonstrated that the aortic valve was edematous despite the absence of apparent vegetative structures, suggesting that the infection mainly involved the aortic ring with MAIVF rather than the aortic valve leaflets. Moreover, an elevated systolic LV pressure due to severe AS might have contributed to fistula formation. Echocardiography is useful for the diagnosis of PMAIVF, although it was unclear whether there was communication between the LVOT and pseudoaneurysm in this case. Additional evaluations using LVG at the outflow tract clarified the communication, which was important to establish the diagnosis of P-MAIVF and to exclude a diagnosis of Valsalva aneurysm $(6,7)$. Xie et al. reviewed 149 cases of P-MAIVF, including seven cases from their institution and 142 cases from published papers (4). Causative organisms included Staphylococcus aureus, different subtypes of streptococci, tubercle bacillus, enterococcus, Brucella suis, Paecilomyces lilacinus, Monilia albicans, and Bacillus spp. According to that review, there were no organism-specific characteristics in the occurrence of P-MAIVF. In the present case, elective surgery was performed, because surgery is the recommended management for P-MAIVF to prevent fatal complications, including compression of coronary arteries or the anterior mitral leaflet and rupture of pseudoaneurysm, which could otherwise lead to cardiac tamponade. We recognized P-MAIVF on hospital day 28, while the operation was performed on hospital day 55. An earlier operation might have been favorable to prevent complications. However, the risk of urgent complication associated with P-MAIVF appeared to be low, as the size of P-MAIVF remained unchanged after detection, and P-MAIVF was unlikely to compress the coronary arteries on MDCT. Therefore, preparation and complete infection control was prioritized before the operation.

According to the operational findings, the tissue around the fistula was discolored, suggesting that the tissue was recently infected. It was likely that the AV node was involved in the infected area. The locational relationship among the fistula, brown discoloration, and AV node was estimated from the operational findings and shown in Fig. 4. According to the reversibility of $\mathrm{AV}$ conduction and the variability of the PQ interval, the most likely site of block was the AV node, rather than the His bundle. It is likely that AV node damage was already present at the time of hospital admission because of the presence of first-degree AV block. In 
cases of AV block due to IE, a decision regarding permanent pacemaker implantation should be considered only after taking into account the possibility that $\mathrm{AV}$ block can resolve spontaneously and the fact that it is not ideal to implant artificial devices at the time of active IE. Interestingly, an AJR was observed during the process of AV block resolution. Although the precise mechanism of the accelerated rhythm is unclear, injury generated by inflammation might have increased junctional automaticity (8).

The authors state that they have no Conflict of Interest (COI).

\section{References}

1. Graupner C, Vilacosta I, San Roman J, et al. Periannular extension of infective endocarditis. J Am Coll Cardiol 39: 1204-1211, 2002.

2. Crimaldi A, Ho SY, Pozzoli A, et al. Pseudoaneurysm of mitralaortic intervalvular fibrosa. Interact Cardiovasc Thorac Surg 13: 142-147, 2011.

3. Park MY, Jeon HK, Shim BJ, et al. Completer atrioventricular block due to infective endocarditis of bicuspid aortic valve. J Cardiovasc Ultrasoud 19: 140-143, 2011.

4. Xie M, Li Y, Cheng TO, et al. Pseudoaneurysm of the mitralaortic intervalvular fibrosa. Int J Cardiol 166: 2-7, 2013.

5. Katayama T, Tsuruya Y, Ishikawa S. Complete atrioventricular block and infective endocarditis in a patient with hypertrophic obstructive cardiomyopathy. Intern Med 51: 749-753, 2012.

6. Hoey ET, Gulati GS, Sihgh S, Watkin RW, Nazir S. The role of multi-modality imaging for sinus of Valsalva aneurysms. Int J Cardiovasc Imaging 28: 1725-1738, 2012.

7. Horimoto K, Kubo T, Matsusaka H, Baba H, Umesue M. Rightsided infective endocarditis with a ruptured sinus of valsalva and multiple septic pulmonary emboli in a patient with atopic dermatitis. Intern Med 54: 797-800, 2015.

8. Sugiyama A, Satoh Y, Ishida Y, Yoneyama M, Yoshida H, Hashimoto K. Pharmacological and electrophysiological characterization of junctional rhythm during radiofrequency catheter ablation of the atrioventricular node. Circ J 66: 696-701, 2002.

The Internal Medicine is an Open Access article distributed under the Creative Commons Attribution-NonCommercial-NoDerivatives 4.0 International License. To view the details of this license, please visit (https://creativecommons.org/licenses/ by-nc-nd/4.0/).

(C) 2016 The Japanese Society of Internal Medicine http://www.naika.or.jp/imonline/index.html 